\title{
Prediction of fatigue crack initiation life of aluminium alloy joints using cyclic elasto-plasticity FEM analysis
}

\author{
Tsutsumi Seiichiro ${ }^{1, *}$, Sano Moe ${ }^{1}$, Fincato Riccardo ${ }^{1}$ \\ ${ }^{1}$ Joining and Welding Research Institute, Osaka University, 11-1 Mihogaoka, Ibaraki, Osaka, JAPAN
}

\begin{abstract}
Finite element analyses (FEA) are particularly useful for investigating fatigue problems since it is possible to carry out elasto-plastic simulations for any configuration and to predict the material behaviour for a large number of loading cycles. This study aims to investigate the fatigue life for Al-Mg alloy A5083$\mathrm{O}$ joints by means of numerical simulations. The Proposed method needs to give a precise description of the elasto-plastic behaviour of the alloy together with an appropriate definition of the criteria for the fatigue crack initiation. In this paper, the elasto-plastic behaviour of the A5083-O alloy was investigated by FE analyses. On the other hand, the fatigue crack initiation criterion is provided based on strain ranges observations. In detail, the finite element analyses focused the attention on the study of the service life of a butt-weld join.
\end{abstract}

\section{Introduction}

Fatigue is one of the main causes of failure of welded structures. Finite element analyses recently became an essential tool for fatigue life estimations, especially when utilizing the advanced fatigue assessment methods for welded structures. The reason for this choice is mainly due to the versatility of the FEM which makes possible to carry out elasto-plastic simulations for any configuration and to predict the material behaviour for a large number of loading cycles.

Fatigue life is usually considered to be divided into two phases: the crack initiation and the crack propagation. Among fatigue failure processes, the prediction of fatigue initiation as a series of damage accumulation and crack initiation is one of the most challenging issues. For the purpose of describing and evaluating the mechanical fatigue processes of materials, a phenomenological plasticity model is proposed [1-4]. This model is based on a cyclic plasticity model [5-9] and has been extended to describe the behaviour of materials, especially steel, and applied to several case studies in low and high cycle fatigue life regions. In this study, we extend the field of investigations to aluminium alloys.

This study aims to investigate the fatigue life for Al$\mathrm{Mg}$ A5083-O aluminium alloy joints by means of numerical simulations. This specific material is very common in the automotive, construction and shipbuilding industries due to the moderately high strength, excellent corrosion resistance and very high toughness, and therefore, the characterization of its fatigue life is crucial to the technological development of the alloy.
In order to characterize the crack initiation life, it is important to give a precise description of the elastoplastic behaviour, which was here investigated by $[1-4$, 10-15]. In addition, the criteria for the fatigue crack initiation was defined based on the strain ranges.

The numerical examples reported in the next sections 3 and 4 show the evaluation of the service life of a buttweld join, modelled with a $2 \mathrm{D}$ geometry under the assumption of a plane strain condition. The model geometry, boundary conditions and material properties were assumed referring to the low-cycle fatigue test carried out by Iida [16]. Three different analyses were carried out in order to investigate the influence of the angular distortion on the service life performances. The fatigue life was judged by observing the local behaviour in terms of plastic strain accumulation and by the definition of a fatigue crack initiation criteria. An investigation in the field of high cycle fatigue $\left(>10^{5}\right)$ was also conducted in the same manner. In this last case, the model geometry was assumed referring to the experimental results carried out by Hirukawa [17].

\section{2 elasto-plastic model}

The elasto-plastic behaviour was investigated with a plasticity model based on the subloading surface model, and extended to include the cyclic damage concepts [14]. A detailed explanation of the model features is beyond the scope of this paper, and the reader is referred to Ref. [1] for a complete discussion. The extended subloading surface model [5-9] describes the generation of plastic strain within the yield surface (subloading surface), which can be obtained through a similarity transformation from the conventional yield surface (referred to as the normal-yield surface in Fig. 1). 
Classical theories distinguish elastic and plastic regions, allowing an irreversible stretch only in the plastic region. In contrast, the subloading surface model abolishes the separation into domains, stating that a plastic response can be realized for every change in the stress state that satisfies the loading criterion. Furthermore, the use of a mobile similarity center, which is a function of the plastic strain, makes this theory particularly suitable for studying cyclic mobility problems.

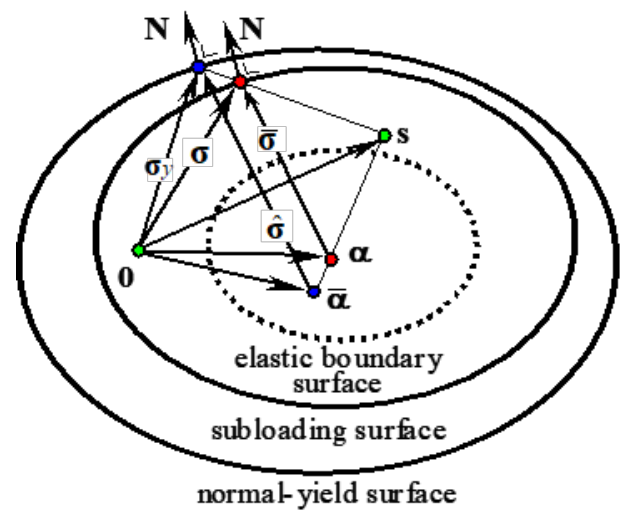

Fig. 1. : Sketch of the Subloading, elastic boundary and normal yield surfaces in the stress space.

Starting from the original subloading surface model a scalar damage variable was introduced to simulate the loss of stiffness induced by the material mechanical degradation. The damage is coupled with a special variable of the cumulative plastic strain $\mathrm{H}_{\mathrm{d}}$ according to the following formula:

$$
D\left(H_{d}\right)=\left(1-d_{2}\right)\left[1+\left(\frac{d_{1}}{H_{d}}\right)^{d_{3}}\right]^{-1}
$$

where $d_{1}, d_{2}$, and $d_{3}$ are material parameters that regulate the damage rate evolution, as shown in Fig. 2, and they must be calibrated through an experimental campaign.

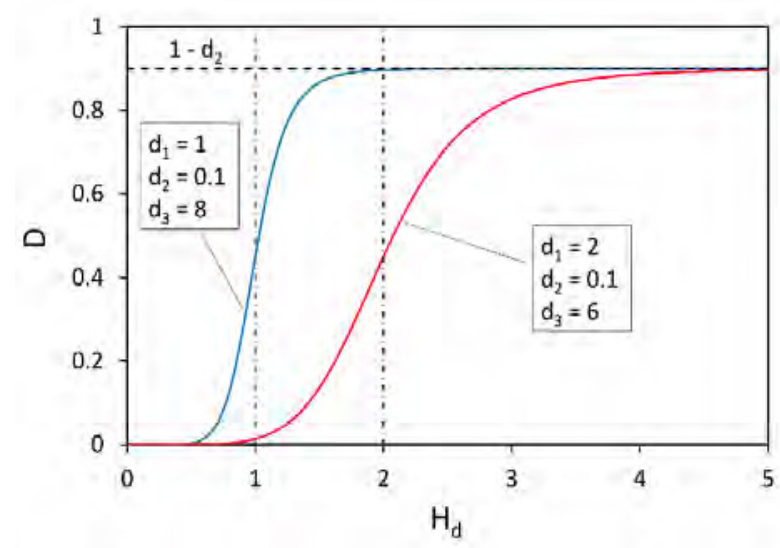

Fig. 1. Damage evolution for two sets of material parameters.

The material hardening behaviour was described by the combination of isotropic and kinematic hardening laws, in Eq.(2) and Eq.(3), respectively.

$$
F=F_{0}\left[1+h_{1}\left\{1-e^{\left(-h_{2} H\right)}\right\}\right], \quad F^{\prime}=F_{0} h_{1} h_{2} e^{\left(h_{2} H\right)}
$$

$$
\boldsymbol{\alpha}=a_{1}\left(a_{2} F \mathbf{N}-\sqrt{\frac{2}{3}} \boldsymbol{\alpha}\right)\left|\varepsilon^{p}\right|
$$

where $\mathrm{h}_{1}, \mathrm{~h}_{2}, a_{1}$ and $a_{2}$ are four material constants defined by the user.

Previous works carried out by the authors [1-4, 1015], focused the attention on steel components; in this study, we extend the field of investigations to the aluminium alloy A5083-O.

The identification of the plastic parameters was carried out through inverse analyses by minimizing the difference between the experimental data found in Horikawa and Cho [18] and the numerical results obtained by the FE analyses. Fig. 3 reports the comparison of the model response against the experimental results, showing a good agreement between the curves. The material parameters used in the computation are reported in the following in Table. 1.

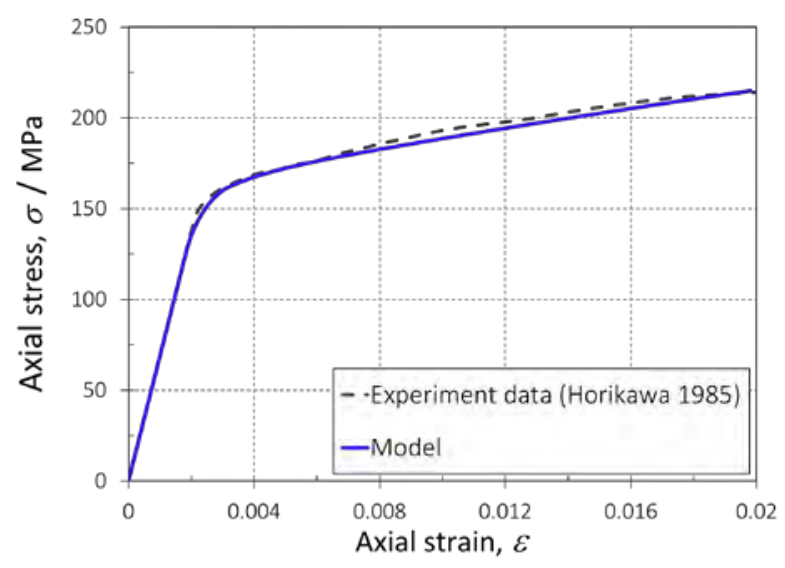

a) Tensile stress-strain curve

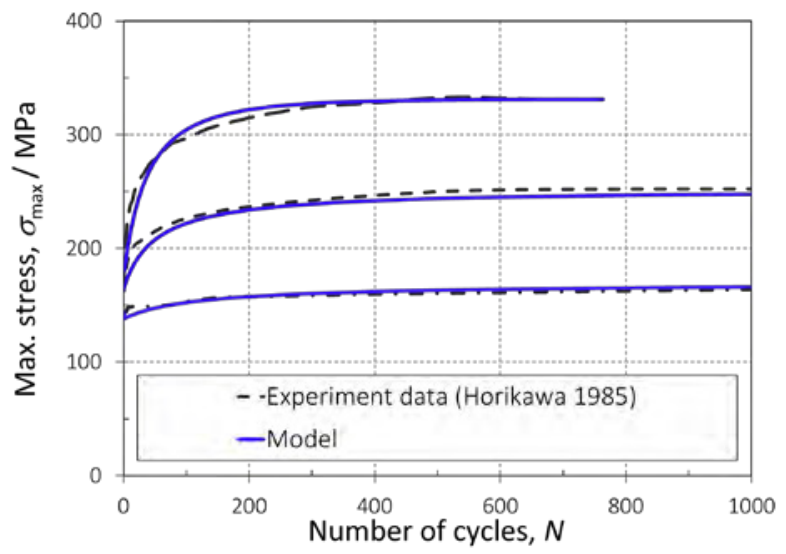

b) Max stress versus number of cycles in the fatigue test (displacement control boundary condition).

Fig. 3. Comparison of the numerical model response against the experimental results. 
Table 1. Material Parameter used in this paper

\begin{tabular}{|c|c|}
\hline Material parameter & value \\
\hline$E$ & $68,600[\mathrm{MPa}]$ \\
\hline$v$ & 0.33 \\
\hline$u$ & 200 \\
\hline$F_{0}$ & 168 \\
\hline$R_{e}$ & 0.4 \\
\hline$h_{1} ; h_{2}$ & $1.05 ; 1.8$ \\
\hline,$a_{a} ; \gamma_{a}$ & $20 ; 0.6$ \\
\hline
\end{tabular}

\section{Fatigue crack initiation criteria}

In order to characterize the fatigue life, it is also important to give an appropriate definition of the criteria for the fatigue crack initiation.

The relation between the strain ranges and fatigue life is defined based on previous experimental data in [16], [18, 19]. The following Eq. (4) was formulated by means of the least square method on all the experimental results, obtaining the solid line in Fig.4:

$$
\frac{\Delta \varepsilon}{2}=14.5 N_{f}^{-0.488}+0.168
$$

where $\Delta \varepsilon$ and $N_{f}$ are the total strain range and the fatigue life, respectively.

In the same manner, the relation between the crack initiation life and fatigue life can be analytically given by the following expression:

$$
N_{c}=0.654 N_{f}
$$

$N_{c}$ is the crack initiation life. In this paper, the surface crack initiation size is defined as a crack of $0.5 \mathrm{~mm}$ length. Finally, combining Eq. (4) and (5), the relation between the total strain ranges and crack initiation life is obtained as follows:

$$
\frac{\Delta \varepsilon}{2}=11.9 N_{f}^{-0.475}+0.168
$$

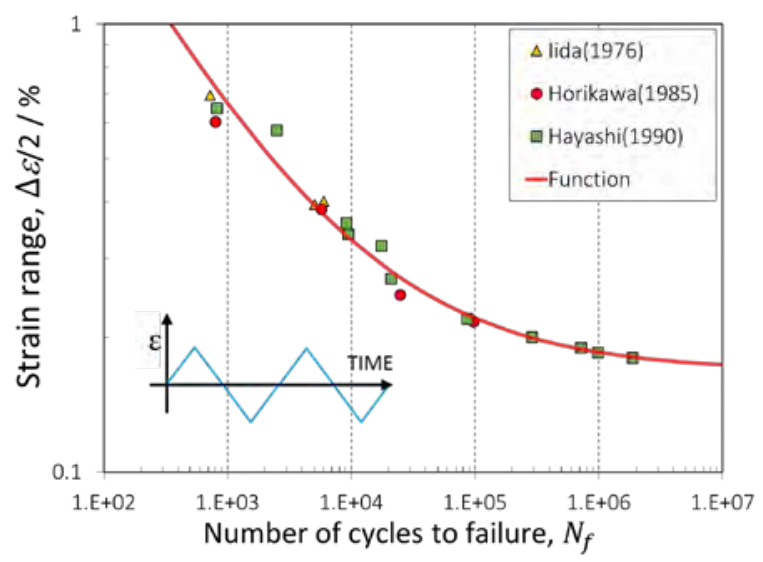

Fig. 4. Strain range vs number of cycles to failure.

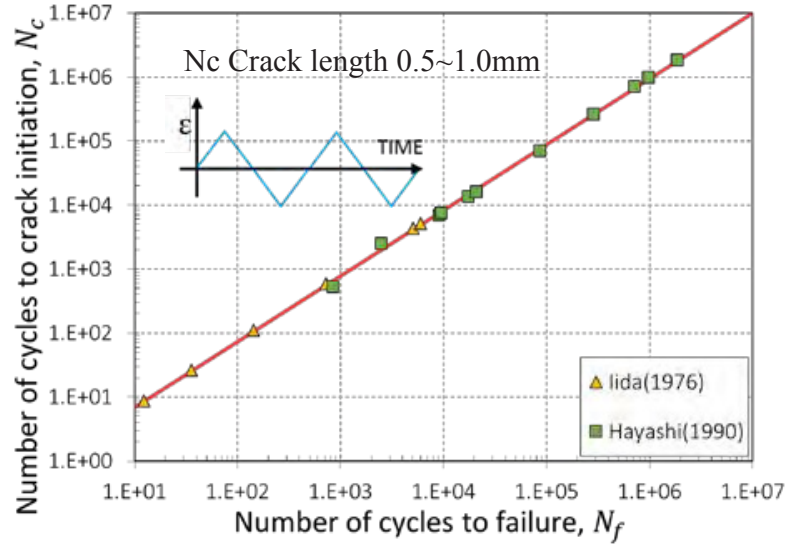

Fig. 5. Crack initiation life vs number of cycles to failure

\section{Analysis of Low cycle fatigue}

\subsection{FE model}

The constitutive equations of the phenomenological model in [1-4] were implemented via user subroutine for the commercial code Abaqus (ver. 6.14-4).

The numerical models were created referring to the experimental results carried out by Iida [16]. The finite element analyses focused the attention on the study of the service life of a butt-weld join, modelled with a 2D geometry under the assumption of a plane strain condition. A mesh refinement $(0.05 \mathrm{~mm}$ minimum element dimension) was performed around the welding toe, where the largest stress concentration tends to appear. A total of 4840 plate elements were used in the discretization, amounting to a total of 5874 nodes. The numerical simulations were conducted for cyclic loading with a constant amplitude (i.e. unidirectional cycling loading $\mathrm{R}=0$ ).

As a result of the welding process, aluminum alloys show higher angle distortions compared to common carbon steels, which impacts significantly to the fatigue life of the components. In this study, three cases were reproduced simulating three different angular distortions; $0^{\circ}, 0.5^{\circ}$ and $1.0^{\circ}$ (see Fig. 7 as reference).

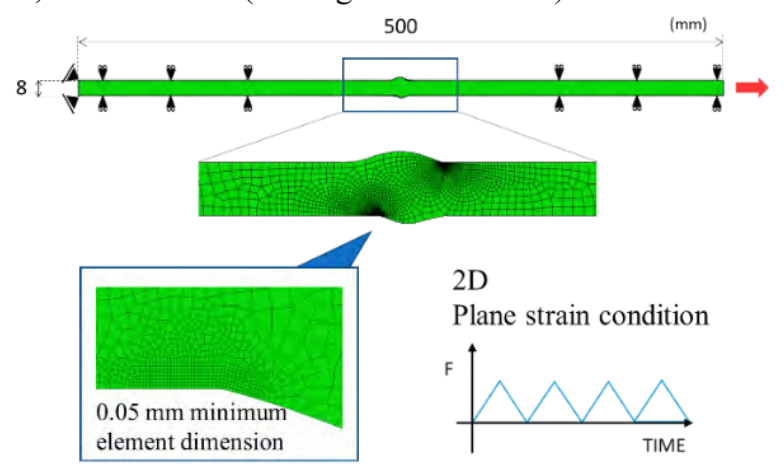

Fig. 6. FE model. 


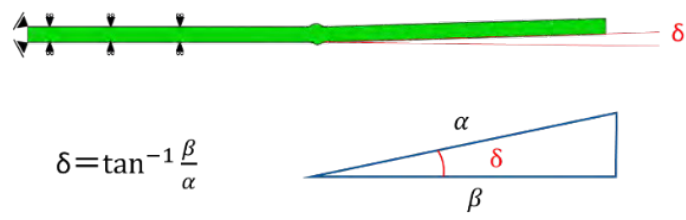

(1) $\delta=0$ Deg. (2) $\delta=0.5 \mathrm{Deg}$. (3) $\delta=1.0 \mathrm{Deg}$.

Fig. 7. Sketch of the angular distortion

\subsection{Result}

Fig. 9 shows the local S-N curves obtained from FE analyses. The local stress-strain response, taken in correspondence of the element which shows a higher plastic accumulation, is used for the life prediction. The result is particularly relevant for the expected fatigue life of the components, as shown in Fig. 10.

The black dashed line indicates the experimental results carried out by Iida [16]. The blue solid line indicates the numerical results of the $0^{\circ}$ angular distortion case. Analogously, the green and red lines represent the $0.5^{\circ}$ and $1.0^{\circ}$ angular distortion cases, respectively. In the low cycle region, the simulations showed good agreement with the experimental results [16], confirming the predicting capability of the numerical model. Whereas, in the high cycle region, the estimated crack initiation life is longer than the experimental results.

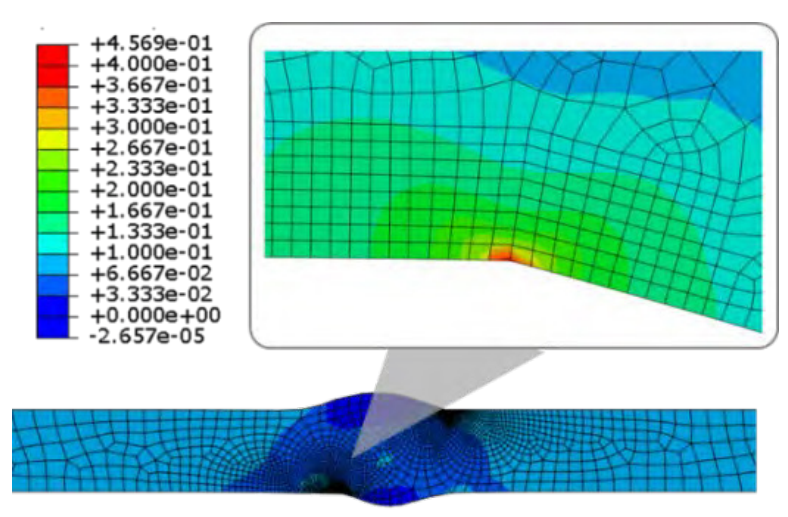

Fig. 8. Cumulative equivalent plastic strain distributions

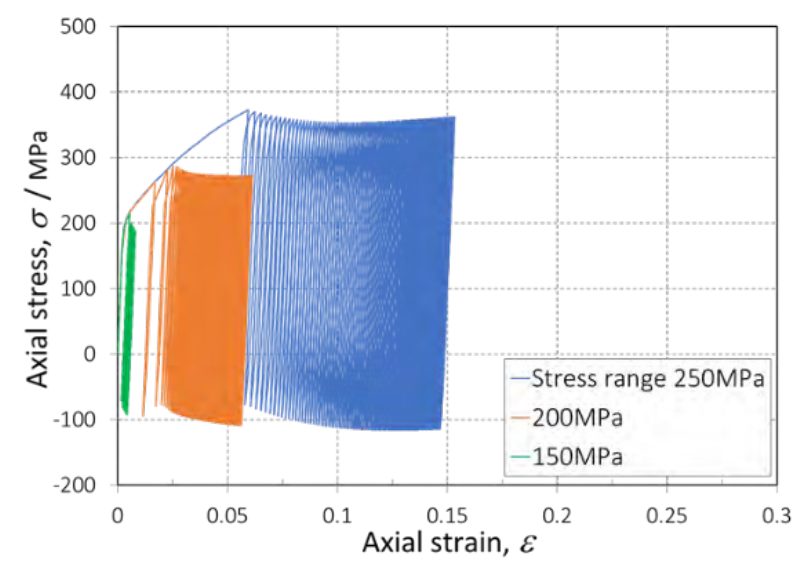

Fig. 9. Predicted local stress-strain curves.

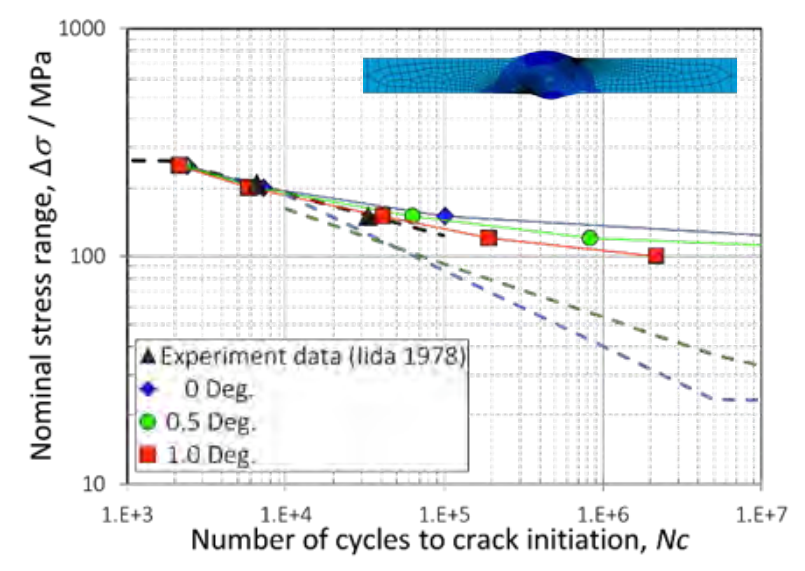

Fig. 10. Predicted S-N curves for crack initiation.

\section{Analysis of High cycle fatigue}

\subsection{FE model}

In this case, the numerical models were created referring to the experimental results carried out by Hirukawa [17]. A butt-weld join was modelled with a 2D geometry under the assumption of a plane strain condition. A mesh refinement ( $0.05 \mathrm{~mm}$ minimum element dimension) was performed around the welding toe. A total of 6400 plate elements were used in the discretization, amounting to a total of 6615 nodes.

The numerical simulations were conducted for cyclic loading with a constant amplitude $(\mathrm{R}=0)$. Here, two cases were reproduced simulating two different angular distortions; $0^{\circ}$, and $1.0^{\circ}$.

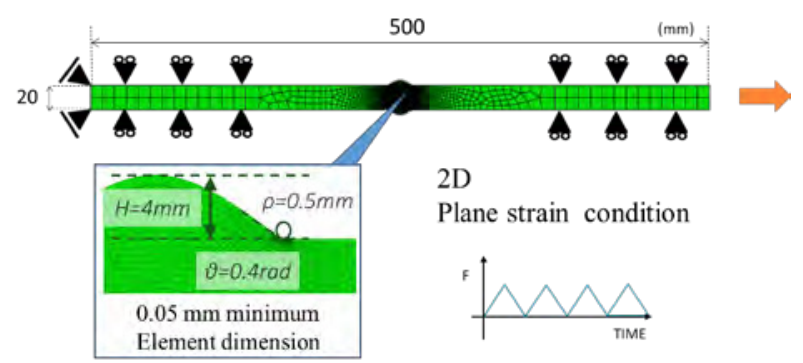

Fig. 12. FE model

\subsection{Fatigue crack propagation analysis}

In this case, fatigue crack propagation analysis was carried out using X-FEM, after predicting crack initiation life. The analysis was carried out using the open-source Salome-Meca software. A linear static analysis is performed based on the assumption of a small scale yielding condition of the fatigue crack. Paris' law is employed as crack propagation law.

$$
\frac{d a}{d N}=C \Delta K^{m}
$$


where $d a / d N$ is crack velocity and $\Delta K$ is the range of stress intensity factor. The material parameters for the Paris' law are presented in Table 2. These parameters were experimental data obtained by Takaai [20].

Table 2. Material parameters of the Paris' law.

\begin{tabular}{|c|c|}
\hline$m$ & 2.7 \\
\hline$C$ & $3.3 \times 10^{-10}$ \\
\hline
\end{tabular}

The model geometry and the predicted crack location were taken from the crack initiation analysis.

\subsection{Results}

Fig. 13 shows the local S-N curves obtained from FE analysis. The local stress-strain response, taken in correspondence of the element which shows a higher plastic accumulation, is used for the crack initiation life prediction. Fig. 14 shows the schematic representation of fatigue crack path that was obtained from the fatigue crack propagation analysis. The fatigue life was obtained by adding the predicted crack initiation life to the propagation life. The result is particularly relevant for the expected fatigue life of the components, as shown in Fig. 15. The black dots indicate the experimental results carried out by Hirukawa [17]. The green solid line indicates the numerical results of the $0^{\circ}$ angular distortion case. Analogously, the blue line represents the $1.0^{\circ}$ angular distortion cases. If the angular distortion is taken into account, the simulations come closer to the experimental results. However, in the low cycle region, the estimated fatigue life is shorter than experimental results. Whereas, in the high cycle region, the estimated one is longer than the experimental results.

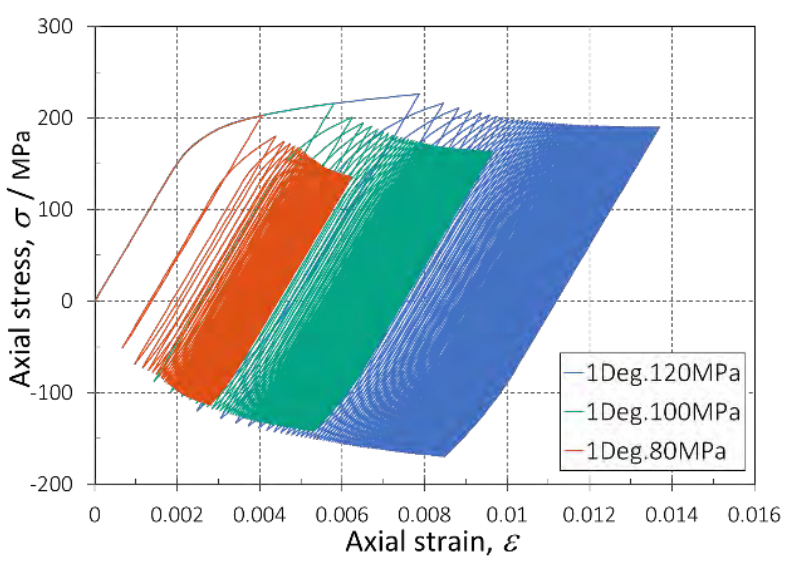

Fig. 13. Predicted local stress-strain responses.

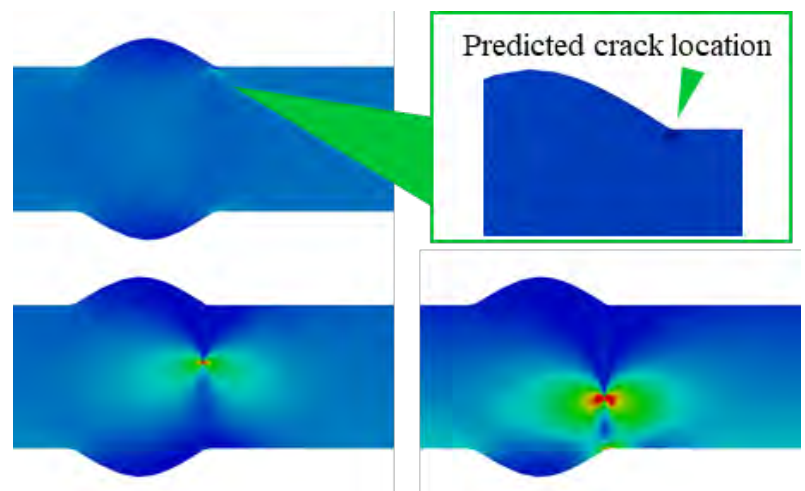

Fig. 14. Schematic representation of fatigue crack path.

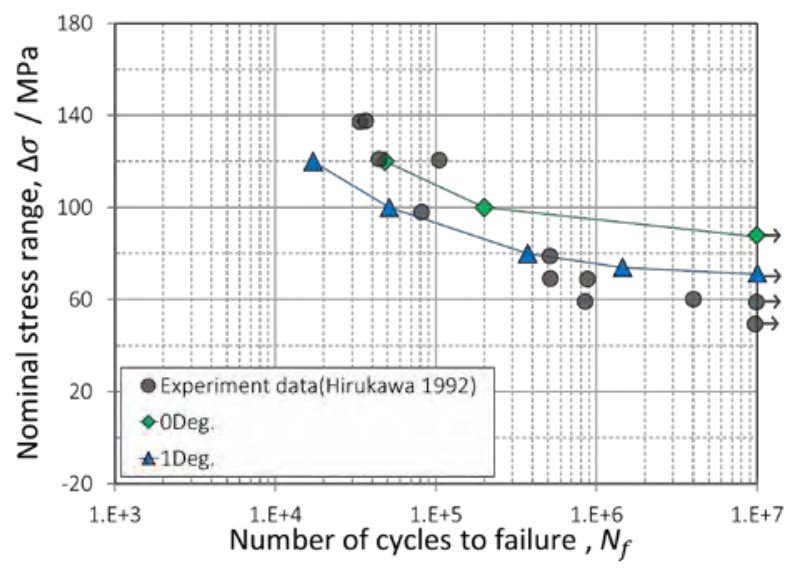

Fig. 15. Predicted S-N curves for total life

\section{Conclusion}

The paper presented a series of investigations on the fatigue crack initiation life for aluminum alloy A5083-O joints, by means of numerical simulations. The work can be summarized in the following points:

- the elasto-plastic behavior of the material was investigated using an unconventional plasticity model;

- a crack initiation criterion was formulated;

- The numerical simulations under uniaxial condition were conducted with constant strain rate in two cases. The first case in low cycle fatigue field, and other in the high cycle fatigue region. The results were compared against the experimental data.

The simulations in the low cycle fatigue region showed good agreement with the experimental results, confirming the predicting capability of the numerical model. However, the estimated fatigue life was longer than the experimental one in the high-cycle fatigue regime.

The simulations in the high cycle fatigue field were conducted using FEM and X-FEM. The fatigue crack propagation life was estimated together with the crack initiation life. The numerical results indicate the same tendency as experimental results. However, in the low 
cycle region, the estimated fatigue life is shorter than experimental results. Whereas, in high cycle region, the estimated one is longer than experimental results.

Further studies are needed in order to widen the application range of the fatigue assessment method.

\section{References}

1. S. Tsutsumi, K. Murakami, K. Goto, M. Toyosada, Journal of the Japan Society of Naval Architects and Ocean Engineers, 7 (2008)

2. S. Tsutsumi, M. Toyosada, K. Hashiguchi, J. of. App. Mech. JSCE, 9, 455 (2006)

3. S. Tsutsumi, M. Toyosada, K. Murakami, Transactions of the Japan Society of Mechanical Engineers Series A, JSME, 73 (2007)

4. S. Tsutsumi, M. Toyosada, F. Dunne, Procedia Engineer, 2(1) (2010)

5. K. Hashiguchi, Int. J. Solids Structures, 25 (1989)

6. K. Hashiguchi, T. Yoshimaru, Int. J. Plast. 11, 347 (1995)

7. K. Hashiguchi, S. Tsutsumi, Int. J Plasticity, 17 (2001)

8. K. Hashiguchi, Elastoplasticity Theory (Springer, Berlin, 2009)

9. R. Fincato, S. Tsutsumi, Fracture and Structural Integrity 38, 231 (2016)

10. S. Tsutsumi, K. Morita, R. Fincato, H. Momii, Fracture and Structural Integrity 38, 240 (2016)

11. R. Fincato, S. Tsutsumi, Marine Structures, 55, (2017)

12. S. Tsutsumi, K. Morita, R. Fincato, Journal of Structural Engineering A, JSCE, 63, (2017)

13. S. Tsutsumi, R. Nagao, R. Fincato, T. Ishikawa, R. Matsumoto, Q. J. Jpn. Weld. Soc., 35 (2017)

14. R. Fincato, S. Tsutsumi, Q. J. Jpn. Weld. Soc. 35, 185s (2017)

15. H. Momii, S. Tsutsumi, R. Fincato, J. of. App. Mech. JSCE, 73, 2 (2017)

16. K. Iida, K. Minida, Y. Kho, Journal of the Society of Naval Architects of Japan, 144 (1978)

17. H. Hirukawa, S. Matsuoka, E. Takeuchi, S. Nishijima, Transactions of the Japan Society of Mechanical Engineers Series A, 58 (1992)

18. K. Horikawa, S. Cho, Transactions of JWRI, 14(2), (1985)

19. M. Hayashi, T. Kuriyama, Proceedings of the Faculty of Engineering of Tokai University 30.1 (1990)

20. T. Takaai, Y. Nakayama, Japan Inst. Light Metal, 42 (1992) 